\title{
Endovascular treatment of brain arteriovenous malformations involving the anterior cerebral artery
}

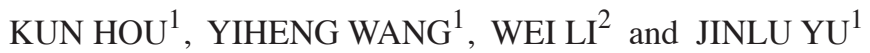 \\ ${ }^{1}$ Department of Neurosurgery, The First Hospital of Jilin University, Changchun, Jilin 130021; ${ }^{2}$ Department of Neurosurgery, \\ The Second Affiliated Hospital of Xingtai Medical College, Xingtai, Hebei 054000, P.R. China
}

Received August 26, 2021; Accepted November 4, 2021

DOI: $10.3892 / \mathrm{mi} .2021 .22$

\begin{abstract}
To date, at least to the best of our knowledge, there are only limited studies available on the endovascular treatment (EVT) of brain arteriovenous malformations (BAVMs) involving the anterior cerebral artery (ACA), thus termed ACA-BAVMs. The present study retrospectively examined 60 patients with ACA-BAVMs treated with EVT. The patients were aged between 10 and 72 years (mean age, 35.4 7.0 years) and included 28 females $(46.7 \%, 28 / 60)$. The ACA-BAVMs were divided into three types: Type I BAVMs were those located below and in front of the corpus callosum genu, type II BAVMs were those located at the upper area of the corpus callosum from the genu to the anterior portion of corpus callosum body, and type III BAVMs were those located from the anterior portion of corpus callosum body to the splenium of the corpus callosum. There were $9(15 \%, 9 / 60), 15(25 \%$, $15 / 60)$ and $36(60 \%, 36 / 60)$ patients with type I, II and III ACA-BAVMs, respectively. Statistical analysis revealed that the posterior cerebral artery (PCA) tended to be involved in type II and III BAVMs. All patients were treated using EVT. During EVT, there were 3 cases of intraoperative bleeding $(5 \%, 3 / 60)$, which tended to occur in type I and II ACA-BAVMs. The other 57 cases $(95 \%, 57 / 60)$ had no complications and new neurological deficits. At the time of discharge, 48 (80\%, 48/60) patients had a Glasgow Outcome Scale score of 5. During the clinical follow-up period, 47 patients were classified according to the modified Rankin Scale (mRS) score; 39 (83\%, 39/47) patients presented with an $\mathrm{mRS}$ score of 0 . On the whole, the
\end{abstract}

Correspondence to: Dr Jinlu Yu, Department of Neurosurgery, The First Hospital of Jilin University, 1 Xinmin Avenue, Changchun, Jilin 130021, P.R. China

E-mail: jlyu@jlu.edu.cn; jinluyu@hotmail.com

Abbreviations: ACA, anterior cerebral artery; BAVM, brain arteriovenous malformation; EVT, endovascular treatment; GOS, Glasgow Outcome Scale; IH, intracerebral hematoma; IVH, intraventricular hemorrhage; mRS, modified Rankin Scale; PCA, posterior cerebral artery

Key words: brain arteriovenous malformation, anterior cerebral artery, endovascular treatment present study demonstrated that EVT may be a safe treatment for ACA-BAVMs.

\section{Introduction}

Brain arteriovenous malformation (BAVM) is a common vascular disease. During the progression of BAVM, the feeding artery may become progressively dilated, thinned and tortuous. The nidus undergoes dynamic remodeling, during the process of which thrombosis or rupture can occur. In addition, the draining vein can become thickened and dilatated due to the continuous high pressure of blood from the feeding arteries $(1,2)$. The typical angioarchitecture of BAVM is illustrated in Fig. 1. However, BAVMs at different sites have different characteristics (3).

BAVMs can occur in the supplying area of the anterior cerebral artery (ACA; thus termed ACA-BAVMs), which is located at the para-midline region of the cerebral hemispheres and is close to the medial veins of the frontal lobe. The posterior part of the ACA is closely associated with the deep cerebral venous system (4). ACA-BAVMs are distributed above the corpus callosum and may be supplied by the A2-A5 segments of the ACA (5).

Currently, endovascular treatment (EVT) is the preferred treatment choice for BAVMs, both as a curative or adjunctive treatment. However, to date, to the best of our knowledge, there are only limited studies available evaluating the safety and efficacy of EVT for ACA-BAVMs. Thus, the present study retrospectively examined the safety of EVT for ACA-BAVMs in a total of 60 patients. The results suggest that EVT may be a safe treatment method for ACA-BAVMs.

\section{Patients and methods}

A total of 60 patients with ACA-BAVMs treated with EVT from January, 2012 to January, 2020 were retrospectively identified at the First Hospital of Jilin University, Changchun, China, and analyzed. The present study was approved by the Ethics Committee of the First Hospital of Jilin University (approval no. 2020-341). Written informed consent was obtained from the patients or their legal relatives.

Inclusion criteria. The inclusion criteria were as follows: i) The BAVM was located above the corpus callosum, at or close to 
the midline of the ACA supplied area; ii) the ACA was the main (if not the only) source of blood supply; iii) the patients had not undergone any EVT, open surgery, or radiosurgery prior to admission to the First Hospital of Jilin University.

ACA-BAVM classification. The ACA-BAVMs were classified according to the anatomical association with the corpus callosum and ACA (Fig. 2). Type I BAVMs were defined as those located at the first segment in Fig. 2, which refers to the area below and in front of the corpus callosum genu (the supplying area of the A2 segment of the ACA). Type II BAVMs were defined as those located at the second segment in Fig. 2, which refers to the upper area of the corpus callosum from the genu to the anterior portion of corpus callosum body (the supplying area of the A3 segment of the ACA). Type III BAVMs were defined as those located at the third segment in Fig. 2, which refers to the area from the anterior portion of corpus callosum body to the splenium of the corpus callosum (the supplying area of the A4-5 segment of the ACA).

Scheme and strategy of EVT. All patients were treated under general anesthesia via a transfemoral approach. Based on the angioarchitecture, the main feeding artery was selected to perform the EVT. For the BAVM nidus, a Marathon microcatheter (Lot no. B143380; product no. 105-5056; Medtronic) or Apollo microcatheter (Lot no. B207314; product no. 105-5096-000; Medtronic) was used to access the nidus of the BAVM to obtain the wedge position. Onyx liquid embolic system (Lot no. B176414; product no. 105-7000-060; Medtronic) was then casted. For flow-related aneurysms, an Echelon microcatheter (Lot no. B229779; product no. 105-5091-150; Medtronic) was preferred to perform coiling.

For thin feeding arteries originating at an acute degree from the ACA in type I and II BAVMs, the micro guide wire could be shaped into a 'J'curve to pass the artery of origin. For type II and III BAVMs with multiple feeding arteries, if satisfactory EVT could not be achieved through the ACA, an additional EVT was performed through other arteries, such as the middle cerebral artery (MCA) and posterior cerebral artery (PCA).

For the EVT of ruptured BAVMs, flow-related aneurysms on the feeding artery or in the nidus of the BAVM were given priority for treatment. In addition, for ruptured BAVMs, if no weak structure was identified, the main purpose of the EVT was to reduce the blood flow of BAVMs. For unruptured BAVMs, the main purpose of the EVT was the targeted embolization of the dangerous structures, such as aneurysms in the feeding artery or dilated structures in the nidus. Conservative management would be recommended for unruptured BAVMs without dangerous structures (6).

Outcome evaluation and follow-up. EVT-related complications, the length of hospital stay and the Glasgow Outcome Scale (GOS) score (1, death; 2, persistent vegetative state; 3, severe disability; 4, moderate disability; 5 , good recovery) at the time of discharge were all recorded (7). The angiographic follow-up and the modified Rankin Scale (mRS) score (0: no symptoms; 1, no significant disability despite symptoms; 2 , slight disability, independent; 3 , moderate disability, requires

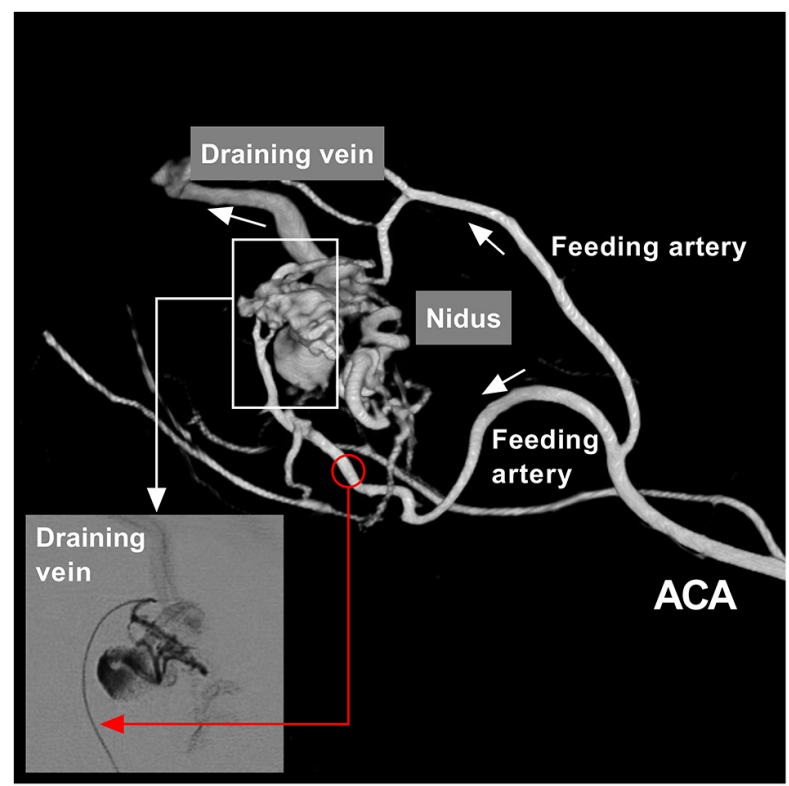

Figure 1. Angioarchitecture of the BAVM. The typical angioarchitecture of the BAVM includes the feeding artery, the nidus and the draining vein. The nidus often contains multiple compartments. The superselective angiogram via a feeding artery (red circle and angled line arrow) indicates a compartment of the BAVM (white rectangle and angled line arrow). ACA, anterior cerebral artery; BAVM, brain arteriovenous malformation.

some assistance, ambulatory; 4, moderately severe disability, requires assistance, not ambulatory; 5, severe disability, bedridden, incontinent, requires constant care) were also recorded (8).

Statistical analysis. GraphPad Software (8.02; GraphPad Software, Inc.) was used for statistical analysis. Continuous variables are expressed as then mean \pm standard deviation. Ordinary one-way ANOVA followed by Tukey's multiple comparisons test was used for the comparison of multiple continuous variables. The Chi-squared test was used to compare count data. A P-value $<0.05$ was considered to indicate a statistically significant difference.

\section{Results}

General patient information. The patients were aged between 10 and 72 years (mean age, $35.4 \pm 17.0$ years), of whom 28 were female $(46.7 \%, 28 / 60)$ and 32 were male $(53.3 \%, 32 / 60)$. A total of $22(36.7 \%, 22 / 60)$ patients had unruptured ACA-BAVMs, of whom 21 patients complained of headaches and 1 patient was admitted for epilepsy. In total, $38(63.3 \%, 38 / 60)$ patients were admitted for intracranial hemorrhage, including 3 patients with subarachnoid hemorrhage, 17 patients with intracerebral hematoma (IH), 1 patient with $\mathrm{IH}$ and subdural hematoma, 4 patients with intraventricular hemorrhage (IVH), and 13 patients with IH and IVH. Of the 38 patients with ruptured BAVMs, 18 patients were classified as Hunt-Hess grade I, 8 as grade II and 12 as grade III (9).

Imaging characteristics. The diameter of the ACA-BAVMs was $4.4 \pm 2.6 \mathrm{~cm}$ (range, $0.75-13.5 \mathrm{~cm}$ ). The size was $<3 \mathrm{~cm}$ in 19 cases $(31.7 \%, 19 / 60), 3-6 \mathrm{~cm}$ in 28 cases $(46.7 \%, 28 / 60)$ and 


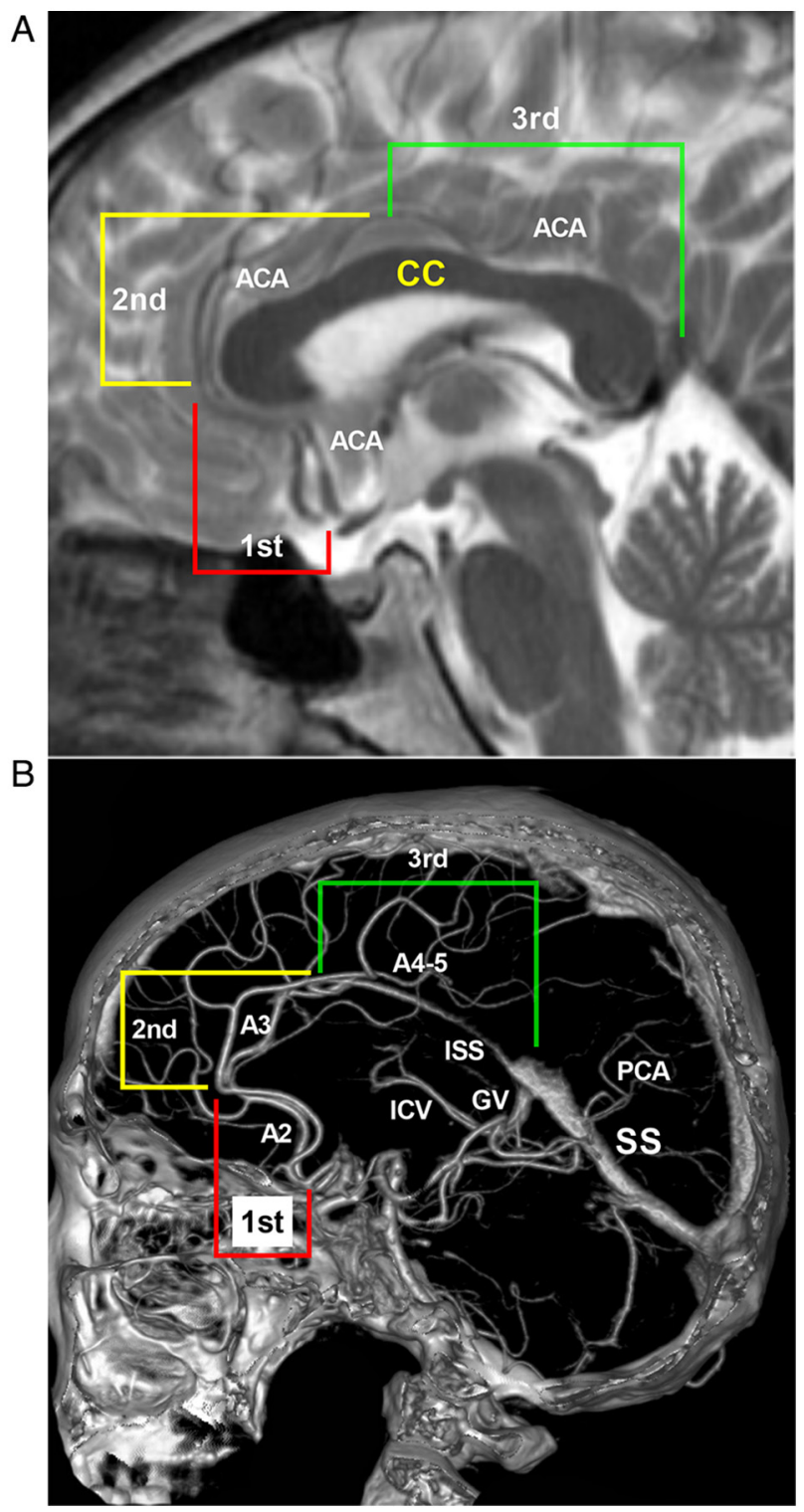

Figure 2. BAVM classification according to the ACA and corpus callosum anatomy. (A) Head MRI illustrating the different types of ACA-BAVMs along the various segments of the CC and ACA. Type I ACA-BAVM locates at the first segment, that is, the anteroinferior part of the $\mathrm{CC}$ genu. Type II ACA-BAVM locates at the second segment, that is, from the genu to the anterior portion of CC body. Type III ACA-BAVM locates at the third segment, that is, from the anterior portion of $\mathrm{CC}$ body to the splenium of the CC. (B) Location of the different types of ACA-BAVMs along the ACA on head CTA (sagittal view). Type I BAVM is supplied by the A2 segment of ACA. Type II BAVM is supplied by the A3 segment of ACA. Type III BAVM is supplied by the A4-5 segment of ACA. ACA, anterior cerebral artery; BAVM, brain arteriovenous malformation; $\mathrm{CC}$, corpus callosum; CTA, computed tomography angiography; GV, Galen vein; ICV, the internal cerebral vein; ISS, inferior sagittal sinus; MRI, magnetic resonance imaging; PCA, posterior cerebral artery; SS, straight sinus.

$>6 \mathrm{~cm}$ in 13 cases $(21.6 \%, 13 / 60)$. In total, 16 cases $(26.7 \%$, $16 / 60$ ) were classified as Spetzler-Martin (SM) grade I, 20 cases $(33.3 \%, 20 / 60)$ as SM grade II, 21 cases $(35 \%, 21 / 60)$ as SM grade III and 3 cases $(5 \%, 3 / 60)$ as SM grade IV (10).

A total of 6 cases $(10 \%, 6 / 60)$ had flow-related aneurysms on the feeding artery. In addition, in 42 cases (70\%, 42/60), the ACA-BAVMs were drained by the superficial veins, in 14 cases $(23.3 \%, 14 / 60)$ they were drained by the deep veins,
Table I. Main clinical data of the patients in the present study.

\begin{tabular}{|c|c|}
\hline Characteristic & Data \\
\hline \multicolumn{2}{|l|}{ Age (years) } \\
\hline Mean \pm SD & $35.4 \pm 17.0$ \\
\hline Range & $10-72$ \\
\hline Female/male & $87.5 \%(28 / 32)$ \\
\hline \multicolumn{2}{|l|}{ Presentation } \\
\hline Unruptured & $22(36.7 \%, 22 / 60)$ \\
\hline Ruptured & $38(63.3 \%, 38 / 60)$ \\
\hline \multicolumn{2}{|l|}{ ACA-BAVM types } \\
\hline I & $9(15 \%, 9 / 60)$ \\
\hline II & $15(25 \%, 15 / 60)$ \\
\hline III & $36(60 \%, 36 / 60)$ \\
\hline \multicolumn{2}{|l|}{ Nidus size (cm) } \\
\hline Mean \pm SD & $4.4 \pm 2.6$ \\
\hline Range & $0.75-13.5$ \\
\hline \multicolumn{2}{|l|}{ Spetzler-Martin grade } \\
\hline 1 & $16(26.7 \%, 16 / 60)$ \\
\hline 2 & $20(33.3 \%, 20 / 60)$ \\
\hline 3 & $21(35 \%, 21 / 60)$ \\
\hline 4 & $3(5 \%, 3 / 60)$ \\
\hline Associated aneurysm & $10(10 \%, 6 / 60)$ \\
\hline \multicolumn{2}{|l|}{ Draining vein } \\
\hline Superficial & $42(70 \%, 42 / 60)$ \\
\hline Deep & $14(23.3 \%, 14 / 60)$ \\
\hline Both & $4(6.7 \%, 4 / 60)$ \\
\hline \multicolumn{2}{|l|}{ Embolization degree of the nidus } \\
\hline Complete or nearly complete & $34(56.7 \%, 34 / 60)$ \\
\hline Partial & $22(36.7 \%, 22 / 60)$ \\
\hline Intact & $4(6.7 \%, 4 / 60)$ \\
\hline \multicolumn{2}{|l|}{ Complications } \\
\hline Intraoperative bleeding & $3(5 \%, 3 / 60)$ \\
\hline \multicolumn{2}{|l|}{$\begin{array}{l}\text { Glasgow Outcome Scale at time } \\
\text { of discharge }\end{array}$} \\
\hline 5 & $48(80 \%, 48 / 60)$ \\
\hline 4 & $3(5 \%, 3 / 60)$ \\
\hline 3 & $8(13.3 \%, 8 / 60)$ \\
\hline 2 & 0 \\
\hline 1 & $1(6.1 \%, 2 / 33)$ \\
\hline
\end{tabular}

ACA, anterior cerebral artery; BAVM, brain arteriovenous malformation.

and in 4 cases $(6.7 \%, 4 / 60)$, they were drained by both the superficial and deep veins.

ACA-BAVM types. Type I BAVMs were identified in $9(15 \%$, 9/60) cases. Among these, five were supplied by the ACA, and four were supplied by the ACA and MCA. Type II BAVMs were identified in 15 cases $(25 \%, 15 / 60)$. Among these, nine were supplied by the branches from the ACA, four were supplied by the ACA and MCA, and two were supplied by 


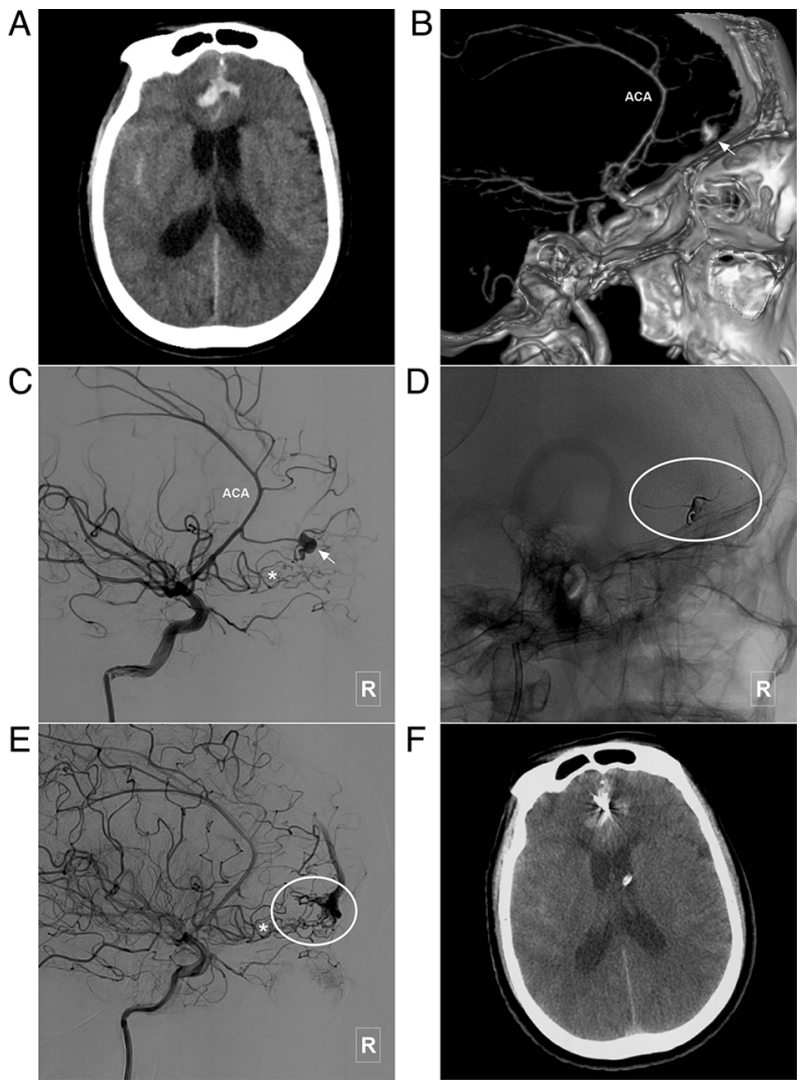

Figure 3. Typical case of a type I ACA-BAVM with flow-related aneurysm. (A) Head CT scan illustrating a subarachnoid hemorrhage in the interhemispheric fissure with hydrocephalus. (B) Head CTA scan illustrating an aneurysm (arrow) located in a branch of the ACA. (C) Angiogram of the right internal carotid artery in lateral view indicates a flow-related aneurysm (arrow) in the feeding artery of an ACA-BAVM. The BAVM is supplied by multiple branches (asterisk) of the ACA. (D) X-ray of the cranium indicating Onyx casting (encircled area) following embolization. (E) Angiogram of the right internal carotid artery after embolization illustrating that the aneurysm in the feeding artery is embolized. There is some remnant of the BAVM (encircle area) and some feeding arteries (asterisk). (F) Post-treatment CT scan illustrating that the patient undergoes external ventricular drainage. $\mathrm{ACA}$, anterior cerebral artery; BAVM, brain arteriovenous malformation; $\mathrm{CT}$, computed tomography; CTA, computed tomography angiography; R, right.

the ACA, MCA and PCA. Type III BAVMs were identified in 36 cases $(60 \%, 36 / 60)$. Among these, 18 were supplied only by the ACA, 11 were supplied by the ACA and PCA, five were supplied by the ACA, MCA and PCA, one was supplied by the ACA and MCA, and one was supplied by the ACA, MCA, PCA and external carotid artery.

Results of EVT. Of the $54(90 \%, 54 / 60)$ ACA-BAVMs without aneurysms, $48(80 \%, 48 / 60)$ were embolized with Onyx via the ACA, two $(3.3 \%, 2 / 60)$ via the ACA and MCA, two $(3.3 \%$, $2 / 60)$ via the ACA and PCA, and one $(1.7 \%, 1 / 60)$ via the MCA and PCA. In 1 case $(1.7 \%, 1 / 60)$ with a diffuse type I BAVM, reducing blood flow was achieved by the coiling of the feeding artery.

Of the $6(10 \%, 6 / 60)$ patients with aneurysms, 1 patient $(1.7 \%, 1 / 60)$ with unruptured aneurysm underwent aneurysm coiling and BAVM embolization with Onyx, 2 patients (3.3\%, $2 / 60)$ with unruptured aneurysms underwent aneurysm and BAVM embolization with Onyx, and 3 patients $(5 \%, 3 / 60)$ with
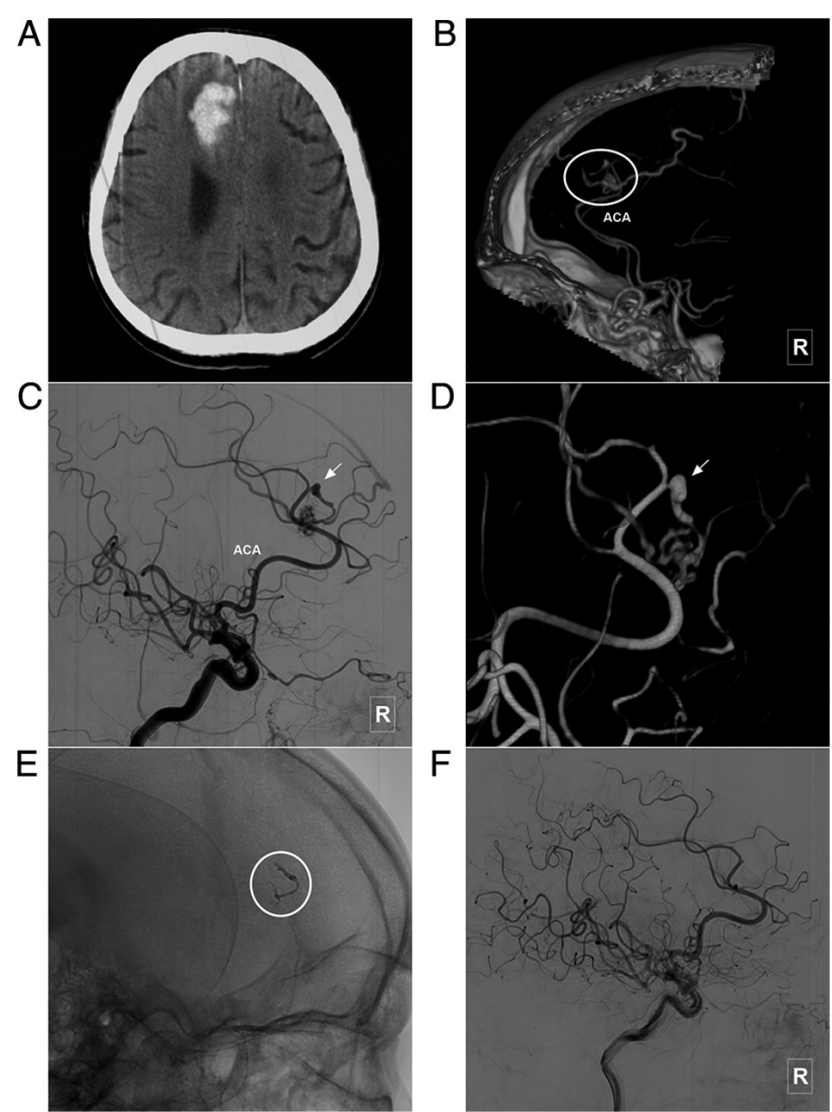

Figure 4. Typical case of a type II BAVM with flow-related aneurysm in the ACA branch. (A) Head CT scan illustrating a hemorrhage in the right frontal lobe. (B) Head CTA scan illustrating a BAVM supplied by the branch of the ACA (encircled area). (C and D) Angiogram of the right internal carotid artery in (C) lateral view and (D) three-dimensional angiogram of the right internal carotid artery indicating that the BAVM is supplied by a single branch of the ACA, with a dissecting aneurysm (arrow) at the beginning of supplying artery. (E) X-ray of the cranium illustrating Onyx casting (encircled area). (F) Angiogram of the right internal carotid artery following treatment indicating the complete embolization of the BAVM and flow-related aneurysm. ACA, anterior cerebral artery; BAVM, brain arteriovenous malformation; CT, computed tomography; CTA, computed tomography angiography; R, right.

ruptured aneurysms underwent coiling only. For the BAVM nidus, immediate complete or nearly complete embolization was achieved in 34 cases $(56.7 \%, 34 / 60)$. Partial embolization was achieved in $22(36.7 \%, 22 / 60)$ cases. Four $(6.7 \%, 4 / 60)$ cases were left untreated (in three cases, after the flow-related aneurysms were embolized, the nidus was treated at a later stage; in one case, the nidus was too diffuse to be embolized, and only the feeding artery was coiled).

During the EVT process, there were 3 cases $(5 \%, 3 / 60)$ of intraoperative bleeding, among whom, 2 cases had type I ACA-BAVMs, and 1 case had type II ACA-BAVMs. When intraoperative bleeding occurred, Onyx casting was continued until termination of the bleeding was achieved. Following EVT, 1 patient died and 2 patients had a favorable recovery.

\section{Outcome and follow-up}

Outcome at discharge. In total, 1 patient $(1.7 \%, 1 / 60)$ died from intraoperative bleeding. At the time of discharge, 8 patients $(13.3 \%, 8 / 60)$ had a GOS score of 3,3 patients $(5 \%, 3 / 60)$ had 

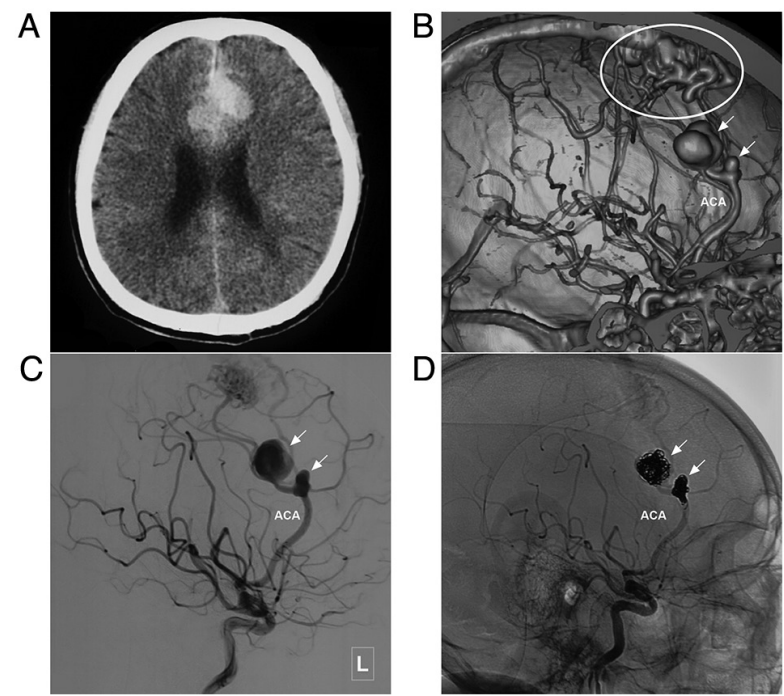

Figure 5. Typical case of a type II BAVM with multiple flow-related aneurysms in the ACA trunk. (A) Head CT scan illustrating a hemorrhage in the interhemispheric fissure. (B) Head CTA illustrating a BAVM (encircled area) supplied by the ACA trunk with multiple flow-related aneurysms (arrows). (C) Angiogram of the left internal carotid artery illustrating that the BAVM is supplied by the ACA trunk with two flow-related aneurysms (arrows). (D) Angiogram of the left internal carotid artery following embolization illustrating that the aneurysms are occluded with coils. ACA, anterior cerebral artery; BAVM, brain arteriovenous malformation; CT, computed tomography; CTA, computed tomography angiography; L, left.

a GOS score of 4 , and 48 patients $(80 \%, 48 / 60)$ had a GOS score of 5 . Of the 59 patients who were discharged, the length of hospital stay ranged from 2-58 days (range, 10.6 \pm 9.3 days).

Outcome at follow-up. A total of $49(81.7 \%, 49 / 60)$ patients could be contacted at the clinical follow-up, which ranged from 12-78 months (mean, $31.1 \pm 20.5$ months). Among these, 2 patients $(4.1 \%, 2 / 49)$ died from non-cerebral disease. Of the remaining $47(95.9 \%, 47 / 49)$ patients, $39(83 \%, 39 / 47)$ presented with an mRS score of $0,3(6.4 \%, 3 / 47)$ with an mRS score of $1,3(6.4 \%, 3 / 47)$ with an mRS score of 2 and $2(4.2 \%, 2 / 47)$ with an mRS score of 3 . In total, $20(33.3 \%$, 20/60) patients underwent an angiographic follow-up, of whom $18(90 \%, 18 / 20)$ had satisfactory embolization and two $(10 \%, 2 / 20)$ underwent additional EVT (after EVT, they had no new neurological deficit). The main clinical characteristics of the patients are presented in Table I. In addition, images of illustrative cases are presented in Figs. 3-8.

Results of statistical analysis. The results of statistical analysis are summarized in Table II. No statistically significant differences were noted in the diameter of the BAVMs, IVH presentation and deep vein drainage among the three types of ACA-BAVMs. Intraoperative hemorrhagic complications tended to occur in type I and II BAVMs (P=0.0223). PCA involvement was more commonly involved in type II and III BAVMs $(\mathrm{P}=0.0052)$.

\section{Discussion}

BAVM is a common congenital vascular disease that is associated with the abnormal connection between arteries and veins, and lacks an intervening capillary network, which can
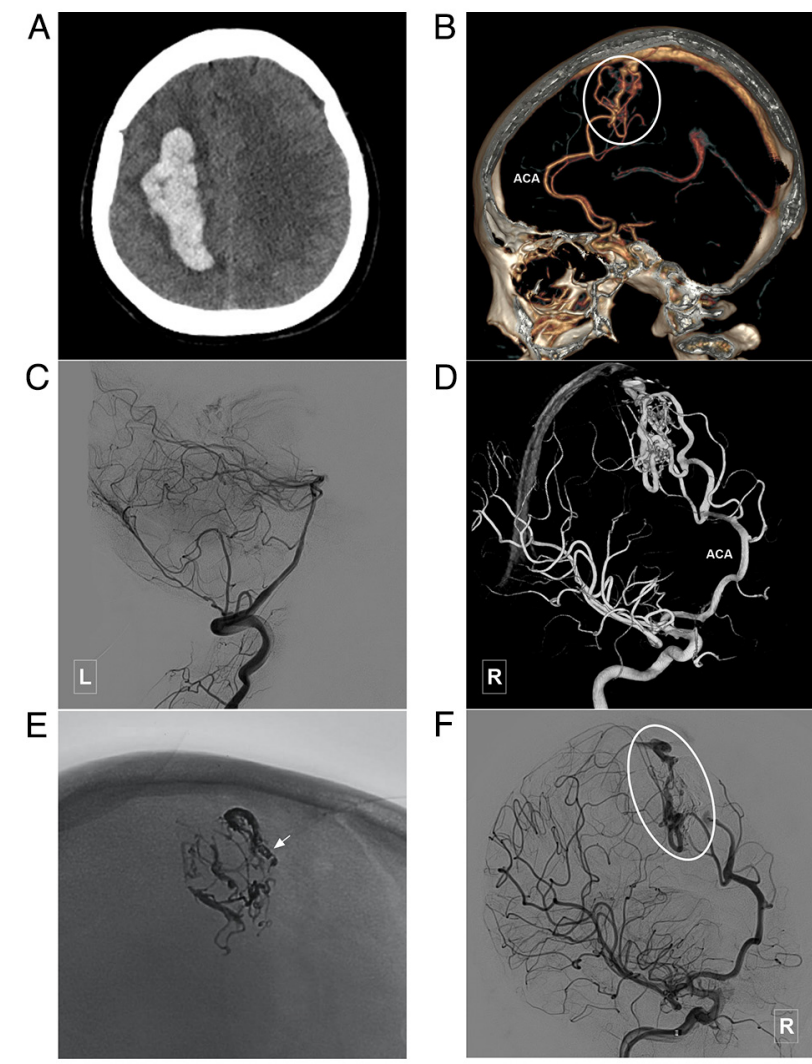

Figure 6. Typical case of a type III BAVM. (A) Head CT scan illustrating a hematoma located in the right fronto-parietal lobe. (B) Head CTA illustrating a BAVM (encircled area) supplied the A4-A5 segment of ACA and drained to the sagittal sinus. (C) Angiogram of the left vertebral artery illustrating that the posterior circulation is not involved in the blood supply of the BAVM. (D) Three-dimensional angiogram of the right internal carotid artery illustrating that the terminal branch of the ACA is involved in the blood supply of the BAVM. (E) X-ray of the cranium illustrating Onyx casting. The arrow denotes the coils in the feeding artery near the nidus, and the "pressure cooker' technique (before Onyx casting, the coiling was performed to occlude the feeding artery to prevent Onyx reflux) is used during treatment. (F) Angiogram of the right internal carotid artery following treatment illustrating that the BAVM is almost completely embolized, while the draining veins (encircled area) remains. ACA, anterior cerebral artery; BAVM, brain arteriovenous malformation; CT, computed tomography; CTA, computed tomography angiography; L, left; R, right.

occur in any part of the brain. The architecture depends on the location, recruited arteries and draining veins, and the size of the BAVM nidus (11).

The ACA lies between the bilateral cerebral hemispheres. It runs an anteroposterior course along the lower part of the cerebral falx and the upper part of the corpus callosum. Under normal conditions, the ACA system is relatively independent (12). In the case of chronic persistent ischemia in the ACA supplying area, the ACA can anastomose with the posterior choroidal arteries from the PCA or with the pial branches of the PCA. This anastomosis is often characterized by a direct anastomosis between the blood supply from the PCA system and the distal trunk of the ACA (13). Generally speaking, the MCA only compensates for the pial branches of ACA.

Some of the BAVMs can occur specifically in the supplying area of ACA, exhibiting a variety of vascular architectures (14). However, to date, to the best of our knowledge, there is no detailed classification of this specific type of BAVM. In the 
Table II. Results of the statistical analysis of the imaging characteristics of the ACA-BAVMs.

\begin{tabular}{|c|c|c|c|c|}
\hline Characteristics & Type I BAVMs $(n=9)$ & Type II BAVMs $(\mathrm{n}=15)$ & Type III BAVMs $(n=36)$ & P-value \\
\hline BAVM diameter (cm) & $4.89 \pm 2.60$ & $4.67 \pm 2.04$ & $4.18 \pm 2.85$ & 0.3830 \\
\hline IVH presentation & $1 / 5^{\mathrm{a}}$ & $4 / 9^{a}$ & $12 / 24^{\mathrm{a}}$ & 0.4708 \\
\hline Deep vein involvement & $2 / 9$ & $5 / 15$ & $11 / 36$ & 0.8420 \\
\hline Hemorrhagic complication & $2 / 9$ & $1 / 15$ & $0 / 36$ & 0.0223 \\
\hline PCA involvement & $0 / 9$ & $2 / 15$ & $17 / 36$ & 0.0052 \\
\hline
\end{tabular}

${ }^{a}$ Denotes the number of cases of ventricular involvement out of the cases with intracranial pressure. ACA, anterior cerebral artery; BAVM, brain arteriovenous malformation; IVH, intraventricular hemorrhage; PCA, posterior cerebral artery.
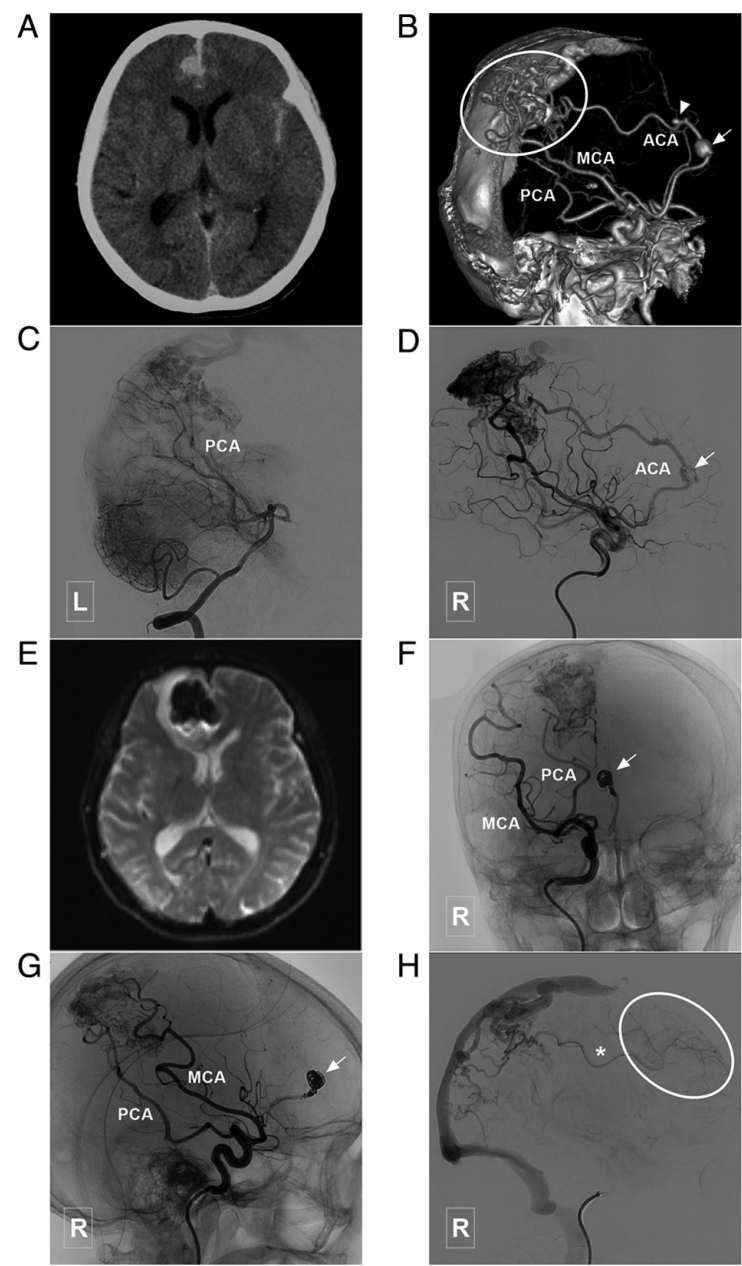

Figure 7. Typical case of a type III BAVM with multiple flow-related aneurysms in the ACA trunk. (A) Head CT scam illustrating a subarachnoid hemorrhage in the interhemispheric fissure. (B) Head CTA illustrating a BAVM (encircled area) supplied the ACA, MCA and PCA, and which drained to the sagittal sinus. The ACA is the main feeding artery, with multiple flow-related aneurysms (arrow and arrow head). (C) Angiogram of the left vertebral artery shows that the posterior circulation is involved in the blood supply of the BAVM. (D) A stent was used to assist in coiling of the aneurysm (arrow) in the ACA. (E) MRI at 3 days post-treatment reveals a new hemorrhage in the interhemispheric fissure, which is considered to be caused by re-rupture of the aneurysm. (F and G) Parent artery occlusion is performed to occlude the aneurysm and the parent artery (arrow). (H) Angiogram of the right internal carotid artery in venous phase illustrating retrograde blood supply from the BAVM to the frontal lobe (encircled area) through the ACA (asterisk). ACA, anterior cerebral artery; BAVM, brain arteriovenous malformation; CT, computed tomography; CTA, computed tomography angiography; L, left; MCA, middle cerebral artery; MRI, magnetic resonance imaging; PCA, posterior cerebral artery; R, right.
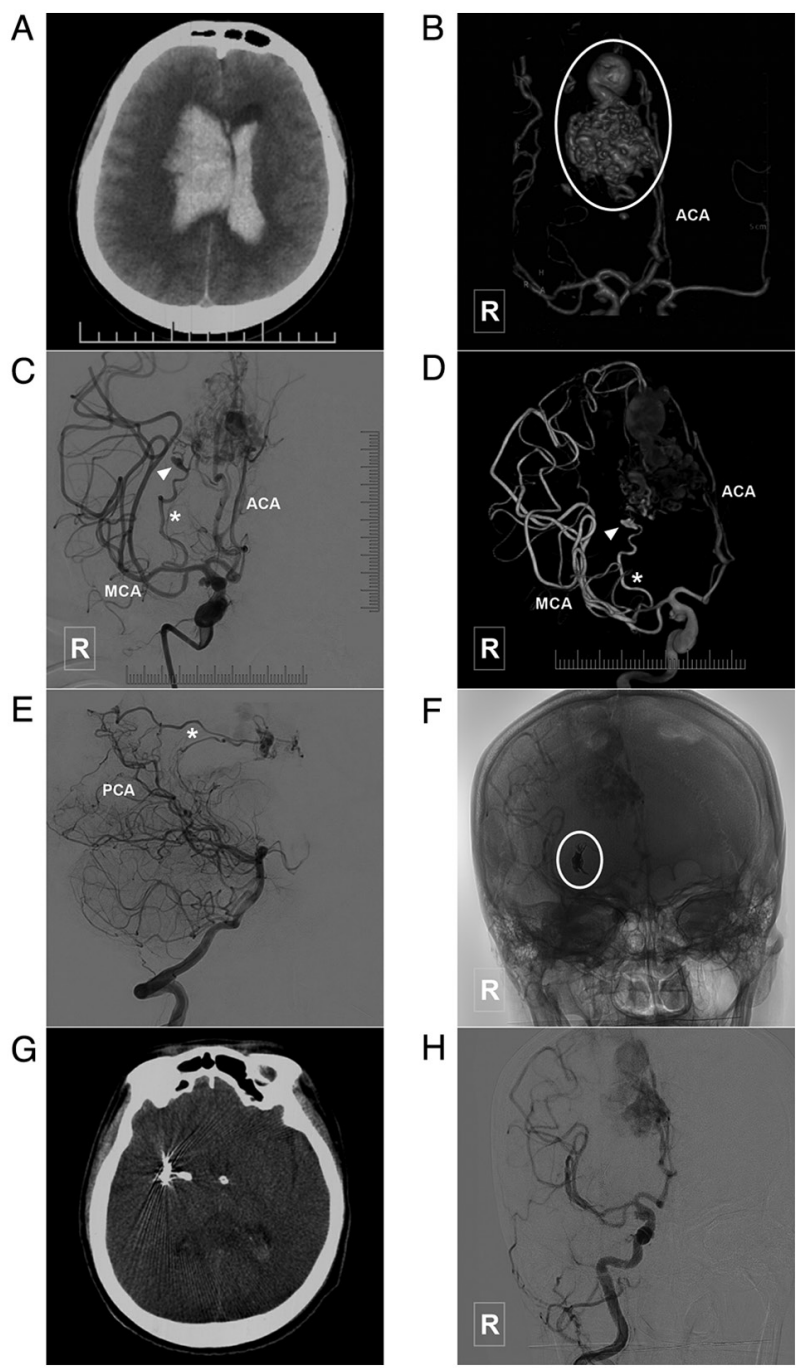

Figure 8. Typical case of a type III BAVM with aneurysm in the lenticular artery. (A) Head CT scan illustrating an intraventricular hemorrhage. (B) Head CTA illustrating a BAVM (encircled area) near the midline. (C and E) Two-dimensional (C and E) and three-dimensional (D) angiogram illustrating a BAVM supplied by the ACA and lenticular artery of the MCA. The asterisk in panels $C$ and D denotes the lenticular artery. The arrowhead denotes the aneurysm in the lenticular artery. Pial collateral circulation from the PCA also supplies the BAVM (asterisk in panel E). (F) Unsubtracted angiogram illustrating that the aneurysm is occluded with coils and Onyx (encircled area). (G) Post-treatment CT scan illustrating external ventricular drainage. $(\mathrm{H})$ Angiogram of the right internal artery 6 months post-treatment showing no recurrence of the aneurysm. ACA, anterior cerebral artery; BAVM, brain arteriovenous malformation; CT, computed tomography; CTA, computed tomography angiography; MCA, middle cerebral artery; PCA, posterior cerebral artery; R, right. 
present study, based on their anatomical association with surrounding structures, the ACA-BAVMs were divided into three types.

In the present study on ACA-BAVMs, the arteries distributed along the ACA differed. For example, there is a potential collateral circulation between the posterior part of the ACA and the PCA, and the anterior part of the ACA can only be compensated by the pial branches from the MCA. BAVMs occurring in the posterior part of the corpus callosum and ACA are closer to the deep venous system (15).

Therefore, statistical analysis was performed for IVH presentation, deep vein involvement and PCA involvement among the different types of ACA-BAVMs to determine whether the types of ACA-BAVMs are associated with IVH presentation, deep vein involvement and PCA. However, the results revealed no significant differences in IVH presentation and deep vein involvement. This suggests that ACA-BAVMs may have the similar imaging and clinical characteristics. However, the statistical analysis revealed that the PCA tended to provide blood flow to the distal ACA-BAVMs.

The principle of EVT for ACA-BAVMs is the same as that for BAVMs in other areas, which mainly aims to manage flow-related aneurysms and the nidus of BAVMs to reduce blood flow to the draining venous system (6). In the present study, 6 cases had with flow-related aneurysms, with an incidence of $10 \%$, which was lower than that observed in a previous study (16). This type of aneurysm is a definite risk factor of BAVMs and requires treatment (Figs. 3, 4, 5, 7 and 8).

The efficacy and safety of EVT for ACA-BAVMs was satisfactory, with a low complication rate of only $5 \%$ in the present study. In the present study, $85 \%$ of the cases had a GOS score of 4 or 5 at the time of discharge, and $89.3 \%$ of cases had an mRS score of 0 and 1 during the follow-up period, which indicated that EVT was a safe strategy for the treatment of ACA-BAVMs.

In the present study, complete or almost complete embolization was achieved in $56.7 \%$ of the cases. The degree of embolization is dependent on a number of factors, such as the size and morphology of the nidus and diameter, and the tortuosity of feeding. Moreover, the degree of embolization is not main treatment goal; the main treatment goal is the management of the weak point posing a risk. Thus, in the present study, the degree of AVM embolization was not pursued.

Following ACA-BAVM classification, EVT becomes strategic. Type I and II BAVMs are supplied by branches of the proximal ACA, and these feeding arteries are commonly slim and multiple. Microcatheter navigation close to the BAVM nidus is difficult, which increases the difficulty in performing EVT and the associated risks. In the present study, intraoperative bleeding tended to occur in patients with type I and II BAVMs. As type II and III BAVMs are supplied by the end of the ACA trunk, performing EVT is relatively simple. However, when they are supplied by multiple regional arteries, particularly from the PCA region, EVT may then be a complex process. In some cases, the PCA has to be used to perform the EVT as the PCA tends to be involved in type II and III BAVMs.

The present study has some limitations which need to be mentioned. One of these limitations is the low rate of angiographic follow-up. The efficacy and safety of EVT were presented as GOS and $\mathrm{mRS}$ scores at the time of discharge and clinical follow-up, which proved that EVT was a safe treatment for ACA-BAVMs. However, due to the very low rate of angiographic follow-up, it is inappropriate to compare the pre- and post-operative angiographic characteristics in this series of patients.

In conclusion, ACA-BAVMs can be classified into types I-III according to the position of the BAVMs along the ACA. This classification is helpful. For type I and II BAVMs supplied by slim and multiple branches of the proximal ACA, EVT is difficult to perform, and intraoperative bleeding complications are common. For type II and III BAVMs, as the PCA tends to be involved, in some cases, the PCA has to be used to perform EVT. On the whole, the present study demonstrated that EVT is a safe procedure for the treatment of ACA-BAVMs.

\section{Acknowledgements}

Not applicable.

\section{Funding}

No funding was received.

\section{Availability of data and materials}

The datasets used and/or analyzed during the current study are available from the corresponding author on reasonable request.

\section{Authors' contributions}

$\mathrm{KH}$ and JY designed the study and drafted the manuscript. $\mathrm{KH}, \mathrm{YW}$ and WL collected the data. KH and JY confirmed the authenticity of all the raw data. YW and WL revised the manuscript. All authors have read and approved the final manuscript.

\section{Ethics approval and consent to participate}

The present study was approved by the Ethics Committee of the First Hospital of Jilin University (approval no. 2020-341). Written informed consent was obtained from the patients their legal relatives.

\section{Patient consent for publication}

The patients or their legal relatives provided consent and agreed for their data (shown in the figures) to be published.

\section{Competing interests}

The authors declare that they have no competing interests.

\section{References}

1. Lawton MT, Rutledge WC, Kim H, Stapf C, Whitehead KJ, Li DY, Krings T, terBrugge $\mathrm{K}$, Kondziolka D, Morgan MK, et al: Brain arteriovenous malformations. Nat Rev Dis Primers 1: 15008, 2015.

2. Solomon RA and Connolly ES Jr: Arteriovenous malformations of the brain. N Engl J Med 376: 1859-1866, 2017. 
3. Ecker RD: Epistemology of brain arteriovenous malformations. World Neurosurg 89: 697-698, 2016.

4. Batista LL and Azevedo HC: Anterior cerebral artery. J Neurosurg 101: 717; author reply 717, 2004.

5. Picard L, Miyachi S, Braun M, Bracard S, Per A and Marchal JC: Arteriovenous malformations of the corpus callosumradioanatomic study and effectiveness of intranidus embolization. Neurol Med Chir (Tokyo) 36: 851-859, 1996.

6. Hou K, Xu K, Chen X, Ji T, Guo Y and Yu J: Targeted endovascular treatment for ruptured brain arteriovenous malformations. Neurosurg Rev 43: 1509-1518, 2020.

7. Jennett B and Bond M: Assessment of outcome after severe brain damage. Lancet 1: 480-484, 1975.

8. Rankin J: Cerebral vascular accidents in patients over the age of 60. II. Prognosis. Scott Med J 2: 200-215, 1957.

9. Hunt WE and Hess RM: Surgical risk as related to time of intervention in the repair of intracranial aneurysms. J Neurosurg 28: 14-20, 1968.

10. Spetzler RF and Martin NA: A proposed grading system for arteriovenous malformations. J Neurosurg 65: 476-483, 1986.

11. Li K, Guo Y, Qu L, Xu B, Xu K and Yu J: Hybrid surgery for an arteriovenous malformation fed by an accessory middle cerebral artery and drained by a developmental venous anomaly: A case report and literature review. Exp Ther Med 16: 1994-2000, 2018.
12. Rhoton AL Jr: The supratentorial arteries. Neurosurgery 51 (Suppl 4): S53-S120, 2002.

13. Hou K, Li G, Luan T, Xu K and Yu J: The prospects and pitfalls in the endovascular treatment of moyamoya disease-associated intracranial aneurysms. Neurosurg Rev 44: 261-271, 2020.

14. Pabaney AH, Ali R, Kole M and Malik GM: Arteriovenous malformations of the corpus callosum: Pooled analysis and systematic review of literature. Surg Neurol Int 7 (Suppl 9): S228-S236, 2016.

15. da Costa MDS, Santos BFO, de Almeida Guardini FB and Chaddad-Neto F: Microsurgical treatment for arteriovenous malformation of the corpus callosum and choroidal fissure. Neurosurg Focus 43: V12, 2017.

16. Cagnazzo F, Brinjikji W and Lanzino G: Arterial aneurysms associated with arteriovenous malformations of the brain: Classification, incidence, risk of hemorrhage, and treatment-a systematic review. Acta Neurochir (Wien) 158: 2095-2104, 2016.

This work is licensed under a Creative Commons Attribution-NonCommercial-NoDerivatives 4.0 International (CC BY-NC-ND 4.0) License. 